\title{
Symptomatic hypothalamic hypopituitarism following radiotherapy
}

\author{
B. M. FRIER* \\ B.Sc., M.B., M.R.C.P. (U.K.) \\ R. J. M. CORRALl \\ B.Sc., M.D., M.R.C.P. (U.K.) \\ Department of Medicine, Western General Hospital, Edinburgh EH4 2XU
}

\begin{abstract}
Summary
A patient is described who developed symptomatic hypopituitarism within 18 months of radiotherapy for nasopharyngeal carcinoma. Detailed investigation indicated a lesion at hypothalamic level. The effect of radiation on hypothalamic-pituitary function is discussed.

\section{Introduction}

The availability of hypothalamic-releasing hormones together with reliable assays of pituitary hormones in plasma have provided the means of accurate assessment of hypothalamic-pituitary function. A case is described of clinical hypopituitarism following radiotherapy to the head and neck, in which the use of these pituitary function tests has demonstrated damage at the hypothalamic level.
\end{abstract}

\section{Case Report}

A 53-year-old postmenopausal woman presented in 1973 with cervical lymphadenopathy, the histology of which was interpreted as an unspecifiable form of malignant lymphoma. She received radiotherapy $(3000 \mathrm{rad})$ to her neck, both axillae and mediastinum. In January 1976 she experienced headaches, diplopia and right-sided facial swelling. Bilateral VIth nerve palsies and sensory impairment over her right lower face were found. She subsequently developed dysphagia, dysarthria and ataxia with cranial nerve palsies of IX, X, XI and XII and bilateral extensor plantar responses. An EMI scan was normal. In September 1976, radiotherapy $(3500 \mathrm{rad})$ was administered to the base of the skull and brain stem area and the neurological deficits improved. Following a further cervical lymph node biopsy in January 1977 the diagnosis was revised to carcinoma of the nasopharynx. Further irradiation was given to the cervical lymph nodes (2500 rad) and nasopharynx $(600 \mathrm{rad})$. She remained weak and unwell with progressive loss of weight. When reinvestigated in November 1977, she was found to be pale and emaciated with normal pubic and axillary hair. The

\footnotetext{
*Present address: Division of Medicine, Royal Infirmary, Edinburgh EH3 9YW.
}

BP was $100 / 80 \mathrm{mmHg}$; plasma sodium was 124 $\mathrm{mmol} / \mathrm{l}$ and chloride $95 \mathrm{mmol} / \mathrm{l}$; haemoglobin was $13.0 \mathrm{~g} / \mathrm{dl}$. The results of pituitary function tests are shown in Table 1. Thyroid antibodies were not detected. Urinary 17-hydroxy-corticosteroid (17OHCS) excretion was $9 \mu \mathrm{mol} / 24 \mathrm{hr}$ (normal 20-50) rising to $91 \mu \mathrm{mol} / 24 \mathrm{hr}$ after 3 daily i.m. injections of $1 \mathrm{mg}$ depot tetracosactrin. Skull $\mathrm{X}$-rays and lateral tomograms of the pituitary fossa showed no evidence of enlargement or erosion of the sella, and visual fields were intact on perimetry.

Replacement therapy with thyroxine and prednisolone resulted in immediate clinical improvement. Her BP increased to $120 / 90 \mathrm{mmHg}$, and plasma electrolytes returned to normal. While previously she had been so weak that she had had difficulty sitting up in bed, within 2 days of starting treatment, she had recovered normal muscle power. Two months after starting replacement therapy, her weight had increased by $5 \mathrm{~kg}$. There was no clinical evidence of persisting hypopituitarism but the serum prolactin level remained markedly elevated at 2240 mu./l, at which time the serum thyroxine concentration (on treatment) was normal.

\section{Discussion}

This patient showed unequivocal evidence of hypopituitarism following radiotherapy for nasopharyngeal carcinoma. The delayed pituitary response to stimulation by TRH (Hall et al., 1972) and LH-RH (Vigersky et al., 1977) and the raised serum prolactin concentration were consistent with a defect at hypothalamic level. The observed rise in urinary 17-OHCS after injections of depot tetracosactrin demonstrated that adrenal hypofunction was secondary to ACTH deficiency. An insulin hypoglycaemia test was not performed because of the patient's poor physical condition.

Pituitary metastases have been reported at postmortem in $25 \%$ of cases with nasopharyngeal carcinoma (Teoh, 1957). This patient, however, had no clinical or radiological evidence of direct involvement of the pituitary fossa or hypothalamus by tumour. In patients with hypopituitarism following 
TABLE 1. Basal plasma hormone levels and the response to stimulation

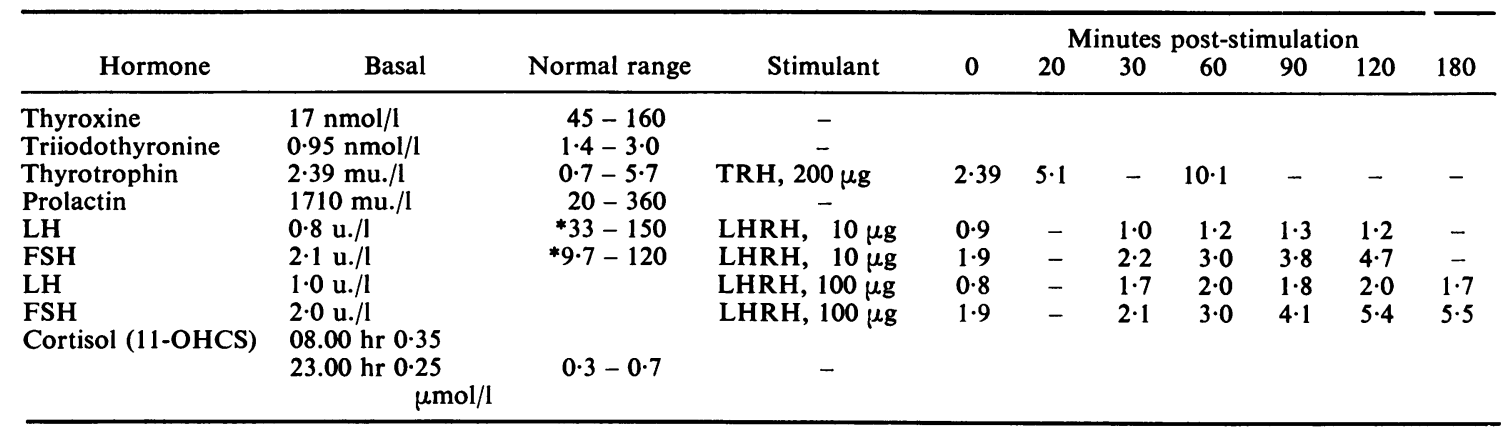

* Values for post-menopausal women.

radiotherapy to the head, radiation damage to the hypothalamus has been implicated as the mechanism of pituitary dysfunction because the hypothalamus is considered more radiosensitive than the pituitary gland (Larkins and Martin, 1973; Samaan et al., 1975; Rosenthal and Goldfine, 1975). This radiosensitivity would explain the hypothalamic hypopituitarism in this case despite the inclusion of the pituitary gland in the field of radiation. Although hypothalamic-pituitary abnormalities have been demonstrated after radiation to the head and neck, very few symptomatic cases of hypopituitarism have been described (Larkins and Martin, 1973; Tan and Kunaratnam, 1966). Previous lack of sensitive hormonal tests of hypothalamic-pituitary function may explain why hypopituitarism has seldom been recognised as a complication of radiotherapy, particularly as the non-specific symptoms of hypopituitarism may be ascribed to the primary malignant disease itself.

Pituitary deficiency has usually been reported as developing several years after radiotherapy (Larkins and Martin, 1973; Samaan et al., 1975). This patient developed hypopituitarism within one year of direct radiation to the nasopharynx, although the hypothalamic-pituitary area was probably irradiated 6 months earlier during treatment of presumed brain stem metastases. The time interval between the radiation and clinical hypopituitarism may therefore be much less than the suggested minimum of 5 years (Samaan et al., 1975).

It seems likely that endocrine abnormalities are a more common complication of radiotherapy to the head and neck than has previously been appreciated (Samaan et al., 1975; Rosenthal and Goldfine, 1976).
The single most useful screening investigation is probably a serum prolactin level (Samaan et al., 1975), elevation of which in this context indicates hypothalamic damage. Measurement of serum prolactin concentrations is therefore advisable before and at regular intervals after radiation of the head, to identify this complication at an early stage. Appropriate replacement therapy, as in this case, may transform the patient's clinical condition.

\section{Acknowledgments}

We thank Dr G. L. Ritchie, Department of Radiotherapy, for permission to report this case, and Professor J. A. Strong for helpful advice.

\section{References}

Hall, R., Ormston, B.J., Besser, G.M., Cryer, R.J. \& MCKENDRICK, M. (1972) The thyrotrophin-releasing hormone test in diseases of the pituitary and hypothalamus. Lancet, i, 759.

LARKINS, R.G. \& MARTIN, F.I.R. (1973) Hypopituitarism after extracranial irradiation: Evidence for hypothalamic origin. British Medical Journal, 1, 152.

Rosenthal, M.B. \& Goldfine, I.D. (1976) Primary and secondary hypothyroidism in nasopharyngeal carcinoma. Journal of the American Medical Association, 236, 1591.

SamaAn, N.A., Bakdash, M.M., CaderaO, J.B., Cangir, A., Jesse, R.H. \& Ballantyne, A.J. (1975) Hypopituitarism after external radiation. Evidence of both hypothalamic and pituitary origin. Annals of Internal Medicine, 83, 771.

TAN, B.C. \& Kunaratnam, N. (1966) Hypopituitary dwarfism following radiotherapy for nasopharyngeal carcinoma. Clinical Radiology, 17, 302.

Tеон, T.B. (1957) Epidermoid carcinoma of the nasopharynx among Chinese: a study of 31 necropsies. Journal of Pathology and Bacteriology, 63, 451.

VigersKy, R.A., ANDERSEN, A.E., ThOMPSON, R.H. \& LORIAUX, D.L. (1977) Hypothalamic dysfunction in secondary amenorrhea associated with simple weight loss. New England Journal of Medicine, 297, 1141. 